\title{
DISERTACIONES
}

ESTUDIOS

Grupos minoritarios y estigmatizados: diversidad funcional, religiosa, étnica, afectivo-sexual o de identidad de género en la comunicación

ISSN: $1856-9536$

Doi: https://doi.org/10.12804/revistas.urosario.edu.co/disertaciones/a.10127

Volumen 15, Número 1 / Enero-junio 2022

Versión PDF para imprimir desde

http://revistas.urosario.edu.co/index.php/disertaciones

Para citar este artículo: Meléndez-Labrador, S. (2022). El lugar de la lengua de señas como lengua minoritaria en la Accesibilidad Comunicativa Universal. Anuario Electrónico de Estudios en Comunicación Social “Disertaciones", 15(1), 1-21. https://doi.org/10.12804/revistas.urosario.edu.co/disertaciones/a.10127

\section{EL LUGAR DE LA LENGUA DE SEÑAS COMO LENGUA MINORITARIA EN LA ACCESIBILIDAD COMUNICATIVA UNIVERSAL}

The Place of Sign Language as a Minority Language in the Universal Communicative Accessibility

O lugar da língua de sinais como língua minoritária na acessibilidade comunicativa universal

Sandra Meléndez-Labrador, Observatorio Latinoamericano de Discapacidad y Comunicación (OBLADIC) ${ }^{1}$

observatorio.obladic@gmail.com

Recibido: 15 de enero del 2021

Aprobado: 20 de mayo del 2021

Fecha de prepublicación: 7 de septiembre del 2021

1 www.obladic.org 


\title{
DISERTACIONES
}

Grupos minoritarios y estigmatizados: diversidad funcional, religiosa, étnica, afectivo-sexual o de identidad de

\section{RESUMEN}

La lengua de señas es reconocida como derecho lingüístico de las personas con discapacidad auditiva de todo el mundo. Entre sus principales características como lengua minoritaria, está la urgencia de su preservación, ya que garantiza el derecho a la información y a la comunicación de la comunidad usuaria. En este sentido, estrategias como las guías y pautas de estilo para la comunicación inclusiva cumplen un papel fundamental en su uso, difusión y accesibilidad. Desde un punto de vista hermenéutico, el presente estudio analiza 22 guías publicadas en español para identificar el lugar que ocupa la lengua de señas como lengua minoritaria en la comunicación inclusiva. Se encontró que la lengua de señas se presenta como una de las formas de expresión de las personas con discapacidad auditiva y como herramienta clave en procesos de participación, lo que permite concluir que estas guías contribuyen en su difusión. El análisis permitió la configuración de un modelo de Accesibilidad Comunicativa Universal que, además de garantizar la preservación de formas alternativas de comunicación de las personas con discapacidad como la lengua de señas, promueve la participación plena de esta población y la autoinclusión.

Palabras clave: lengua de señas; lenguas minoritarias; comunicación; discapacidad; Accesibilidad Comunicativa Universal.

\begin{abstract}
Sign language is recognized as a linguistic right of hearing impaired people all over the world. Among its main characteristics as a minority language is the urgency of its preservation, since it guarantees the right to information and communication of the user community. In this sense, strategies such as style guides and guidelines for inclusive communication play a fundamental role in its use, dissemination and accessibility. From a hermeneutical perspective, this study analyzes 22 guides published in Spanish to identify the place of sign language as a minority language in inclusive communication. It was found that sign language is presented as one of the forms of expression of people with hearing disabilities and as a key tool in participation processes, which allows us to conclude that these guides contribute to its dissemination. The analysis allowed the configuration of a model of Universal Communicative Accessibility that, besides guaranteeing the preservation of alternative forms of communication for people with disabilities such as sign language, promotes the full participation of this population and self-inclusion.
\end{abstract}

Keywords: Sign language; minority languages; communication; disability; Universal Communicative Accessibility.

\section{RESUMO}

A linguagem de sinais é reconhecida como uma linguagem adequada para pessoas com deficiência auditiva em todo o mundo. Entre suas principais características como língua minoritária, está a urgência de sua preservação, uma vez que garante o direito à informação e comunicação da comunidade usuária. Nesse sentido, estratégias como guias de estilo e diretrizes para a comunicação inclusiva desempenham um papel fundamental na sua 


\section{DISERTACIONES}

Grupos minoritarios y estigmatizados: diversidad funcional, religiosa, étnica, afectivo-sexual o de identidad de

utilização, divulgação e acessibilidade. Do ponto de vista hermenêutico, este estudo analisa 22 guias publicadas em espanhol para identificar o lugar da língua de sinais como língua minoritária na comunicação inclusiva. Constatou-se que a língua de sinais, se apresenta como uma das formas de expressão das pessoas com deficiência auditiva, e, como ferramenta fundamental nos processos de participação, o que permite concluir que essas guias contribuem para a sua divulgação. A análise permitiu configurar um modelo de Acessibilidade Comunicativa Universal que, além de garantir a preservação de formas alternativas de comunicação para as pessoas com deficiência, como a língua de sinais, promove a participação plena dessa população e a autoinclusão.

Palavras-chave: língua de sinais; línguas minoritárias; comunicação; deficiência; Acessibilidade Comunicativa Universal.

\section{La lengua de señas como lengua minoritaria}

Según la oms cerca del $5 \%$ de la población mundial posee pérdida de audición discapacitante. Estos 360 millones de personas pueden llegar a comunicarse con los demás a partir de home sign o señas caseras, gestos y lengua de señas ${ }^{2}$ (en adelante Ls). Mientras las Ls se caracterizan por su sistematicidad y convencionalización para ser usados por un número considerado de personas, las señas caseras son un sistema de comunicación básico creado en una familia con uno o pocos miembros sordos, que posee diferencias estructurales importantes con respecto a los otros dos (Meir et al., 2010). Existen 121 tipos de Ls usadas por comunidades de personas sordas ${ }^{3}$ (en adelante PS) que han sido identificadas y tituladas alrededor del mundo, pero pueden superar las 700 (Harrison, 2008). En este sentido, la ps hace parte de una comunidad que es minoría lingüística y cultural.

En general, la diferencia entre los tres sistemas de comunicación de la población sorda radica en el número de personas para quienes resulta ser primario, y es esta diferencia la que permite o no avances, en términos de disposiciones legales que garanticen la vitalidad de estas formas de comunicación, pues lenguas de población minoritaria como personas con discapacidad auditiva, inmigrantes e indígenas, han sido escasamente tenidas en cuenta en derechos humanos lingüísticos en la mayoría de países (Grinevald, 1998; Ahmad, 2008).

2 Según Ortiz (2005), la "lengua de señas" se diferencia del "lenguaje" en que la primera es un sistema lingüístico plenamente definido, además de ser el término utilizado oficialmente por países hispanoamericanos para referirse a la lengua de la comunidad sorda, a diferencia de España, donde se habla de "lengua de signos" de manera equiparable.

3 Las personas sordas hacen parte de una comunidad lingüística que usa la lengua de señas. 


\section{DISERTACIONES}

\section{ESTUDIOS}

En Barcelona, en junio de 1996, 61 Organizaciones No Gubernamentales, 41 Centros PEN (Asociación mundial de escritores) y 40 expertos en derecho lingüístico de todo el mundo se dieron cita para redactar y firmar la Declaración Universal de Derechos Lingüísticos que, aunque no explícita a la ts en su texto, proclama la igualdad sin distinción entre las lenguas, considerando inseparables e interdependientes las dimensiones colectiva e individual de los derechos lingüísticos:

ya que la lengua se constituye colectivamente en el seno de una comunidad y es también en el seno de esta comunidad que las personas usan la lengua individualmente. De esta manera, el ejercicio de los derechos lingüísticos individuales solo puede ser efectivo si se respetan los derechos colectivos de todas las comunidades y grupos lingüísticos. (i Mimó, 1997, p. 12)

De manera más puntual, la onu (2006) estableció la Convención sobre los Derechos de las Personas con Discapacidad (en adelante Convención), en la que instó a "reconocer y promover la utilización de lenguas de señas" (p. 17), así como
b) Facilitar el aprendizaje de la lengua de señas y la promoción de la identidad lingüística de las personas sordas.
c) Asegurar que la educación de las personas, y en particular los niños y las niñas ciegos, sordos o sor- dociegos se imparta en los lenguajes y los modos y medios de comunicación más apropiados para cada persona y en entornos que permitan alcanzar su máximo desarrollo académico y social. (2006, p. 20)

Con respecto al numeral $b$, hay que tener en cuenta que en la mayoría de países existe una única Ls, por ejemplo, la Lengua de Señas colombiana, excepto en Bélgica, Reino Unido, Estados Unidos, India y Tailandia (donde tienen al menos 6 diferentes Ls) (Harrison, 2008) y más recientemente en España, con la Lengua de signos española y catalana.

Más adelante, en el 2016, se presenta el Protocolo para la Garantía de los Derechos Lingüísticos en San Sebastián, el cual dispone medidas esenciales para garantizar los derechos lingüísticos de todo grupo o comunidad, tras el debido consenso entre expertos y agentes que trabajan alrededor de las lenguas y los derechos humanos. En todo caso, como afirman Esteban y Ramallo (2019): "Todas las lenguas de signos están doblemente discriminadas: por un lado, con respecto a las lenguas orales; por otro, su nivel de reconocimiento, protección y promoción está lejos de alcanzar el de muchas lenguas orales consideradas minoritarias” (p. 27).

\section{La supervivencia de la lengua de señas}

El numeral c de la Convención es de vital importancia, ya que a través de la educación se garantiza la supervivencia de una lengua. Dicho proceso de alfabetización debe tener en cuenta las características estructurales que son factores que afectan la vitalidad de las Ls (Bickford et al., 2015), pues se trata de estructuras lingüísticas complejas y distintivas en los aspectos fonéticos, fonológicos, prosódicos, morfológicos y sintácticos, como se pueden consultar en detalle en manuales como el de Pfau et al. (2012) y Hermann y Steinbach (2013). 


\section{DISERTACIONES}

En un primer lugar es necesario garantizar a las PS y personas con discapacidad auditiva (en adelante PCDA) ${ }^{4}$ el derecho a la educación, pues más del $80 \%$ de las personas con discapacidad (en adelante PCD) viven en condiciones de pobreza (onu, 2015), y como aseguran Vico y Heras (2017), a menudo se sigue negando el derecho de aprender y ser usuario de la ss en el ámbito educativo. Teniendo en cuenta lo anterior, la Ls puede no ser ${ }^{5}$ una lengua materna para la mayoría de las personas sordas que se pueden valer de señas caseras en sus contextos familiares, entonces, el aprendizaje de la ss como una segunda lengua puede ser una necesidad predominante en este grupo poblacional. En ese orden, dependiendo de las condiciones sociales, económicas, políticas y culturales de la ps, puede llegar a aprender la ss o la lengua nativa (español) como segunda lengua en un momento tardío de su vida.

Una vez garantizada la inclusión educativa, el periodo crítico de edad es otro factor que interviene de manera crucial tanto en el proceso de aprendizaje de un primer idioma como de un segundo (Johnson \& Newport, 1989). Asimismo, factores como la calidad de la educación en segunda lengua y las similitudes lingüísticas entre el primer y segundo idioma son trascendentales en la adquisición de un lenguaje por parte de alumnos mayores, por ejemplo, una primera lengua tardía como la ss y una segunda lengua como el español (Mayberry, 2010; Hilger et al., 2015). Con respecto a las similitudes lingüísticas, Harrison (2008) desmitifica creencias como que (1) existe una versión del lenguaje local con una seña de mano para cada palabra, por ejemplo, la American Sign Language (ASL) no se parece al inglés en palabras, estructura o reglas gramaticales como puede llegar a serlo la Japanese Sign Language (JsL) con respecto al japonés; y (2) que los signos pueden ser universales para todas las personas sordas, pues en el caso de ASL y JSL no tienen lenguaje en común, sus palabras en seña pueden llegar a ser tan abrumadoramente abstractas que los usuarios de una no pueden entender lo que se trata de decir con la otra lengua.

En resumen, las opciones disponibles de educación para personas sordas pueden variar frente a otros tipos de educación bilingüe, según Baker (2007) citado en Plaza (2012) en las siguientes dimensiones: (a) estatus de lenguaje minoritario (LS) vs. mayoritario (español); (b) competencias del lenguaje previstas (bilingüismo completo o competencia en el lenguaje mayoritario); (c) colocación (segregación vs. mainstreaming o transversalidad); (d) antecedentes inscritos en el lenguaje de los niños; y (e) asignación de los lenguajes en el currículum. Aunque también se puede tener en cuenta el aprendizaje informal que se da de manera intergeneracional (Adam, 2015). En este sentido, vale la pena resaltar el caso de éxito de la educación inclusiva para el alumnado sordo en las últimas décadas, a partir de diversas propuestas o iniciativas bilingües/biculturales en diferentes comunidades autónomas del territorio español presentadas por Vico y Heras (2017).

\section{Mientras unas se extinguen, otras emergen}

Austin y Sallabank (2011) aseguran que muchas de las Ls están siendo documentadas por investigadores como lenguas en peligro de extinción, revelando importantes pistas sobre cómo los seres humanos se comunican en formas no orales. Adicionalmente, los desafíos continúan en la documentación, descripción, preservación y revitalización de casos como las us indígenas en Tailandia (Nonaka, 2009); la lengua de señas rural de campesinos en

4 Se hace referencia a las personas con discapacidad auditiva en general, tanto personas sordas como personas con algún tipo de limitación auditiva.

5 Vale aclarar que existe una minoría de personas sordas, cuyos padres son sordos y adquieren la lengua de señas en el contexto familiar como lengua materna. 


\section{DISERTACIONES}

ESTUDIOS

Ghana, Israel, Tailandia, Marruecos, México, India, Canadá (Esteban \& Ramallo, 2019) o Turquía: ${ }^{6}$ la Mardin Sign Language (MArsL) (Zeshan \& Dikyuva, 2013); o el Hungarian Sign Language que, para el 2006 aún no era tenida en cuenta como lengua oficial en Hungría.

Por otro lado, según Meir et al. (2010), las nuevas lenguas de señas que los lingüistas han comenzado a estudiar en la última década han sido categorizadas como Village Sign Languages (vsL) y Deaf Community Sign Languages (DCSL), las cuales se generan en dos tipos diferentes de ambientes: la primera se desarrolla dentro de pequeñas comunidades o villas y se transmite entre familias cuyas condiciones sociales son determinantes para la estructura lingüística; la segunda, se da en lugares como ciudades y escuelas entre personas con distintos antecedentes, provenientes de diferentes contextos, donde su aprendizaje se da en gran medida entre pares. Ejemplos de vLS son Al-Sayyid Bedouin SL (ABSL, Israel), Martha's Vineyard sL (Estados Unidos), Ban Khor sL (Tailandia), Kata Kolok sL (Bali) y Adamarobe sL (Ghana); y de dcsL figuran como nuevas o modernas: Nicaraguan sL e Israeli sL, con menos de 80 años de aparición.

También están los casos de ss emergentes en lugares de trabajo, como el caso de Lizeth Arce, una joven con discapacidad auditiva que compiló 350 términos gastronómicos en señas que se comparten en varias regiones de Colombia, para que otras personas sordas puedan aprender este campo. Otro ejemplo es el de Meléndez-Labrador (2016), quien en una empresa multinacional identificó que se pueden generar espontáneamente señas diferentes o similares para un mismo producto, dependiendo del contexto cultural en el que se encuentren las sucursales de la franquicia. ¿Podría en estos casos hablarse de lengua de señas laboral?

\section{Accesibilidad Comunicativa Universal}

En línea con la preservación de la Ls, como lengua de una población minoritaria con diversidad funcional, se encuentran las estrategias de uso y difusión de la us como elemento de inclusión y accesibilidad de las ps. Esto se logra a través de lo que la Convención (2006) llama accesibilidad a la información y las comunicaciones. Específicamente el Artículo 2 define:

\section{La "comunicación" incluirá los lenguajes, la visualización de textos, el Braille, la comunicación táctil, los macrotipos, los dispositivos multimedia de fácil acceso, así como el lenguaje escrito, los sistemas auditi- vos, el lenguaje sencillo, los medios de voz digitalizada y otros modos, medios y formatos aumentativos o alternativos de comunicación, incluida la tecnología de la información y las comunicaciones de fácil acceso. Por "lenguaje" se entenderá tanto el lenguaje oral como la lengua de señas y otras formas de comunicación no verbal. (p. 24)}

Entonces, la accesibilidad se trata de identificar y eliminar los obstáculos y barreras de acceso, entre otros, a los servicios de información y comunicaciones, "a fin de que las personas con discapacidad puedan vivir en forma independiente y participar plenamente en todos los aspectos de la vida" (2006, art. 9). En este punto cabe resaltar que la persona sorda, término que representa una reivindicación política que hace alusión a los derechos lingüísticos, es etiquetada con el término "discapacidad" (como persona con discapacidad auditiva) en los procesos de

6 Donde la pérdida de audición es una situación hereditaria durante muchas generaciones llevándolos a consolidar una comunidad de signos compartida. 


\section{DISERTACIONES}

accesibilidad para referirse a las barreras comunicativas desde dicho paradigma, minorizando así la perspectiva antropológica e identitaria de las personas sordas (Esteban \& Ramallo, 2019), por lo tanto, en adelante se usarán ambos términos: personas sordas/personas con discapacidad auditiva (PS/PCDA).

En este orden, se propone para el presente estudio desarrollar el concepto de Accesibilidad Comunicativa Universal, con el que se busca ir más allá de los modos, medios y formatos de comunicación accesible, es decir, que permiten meramente el acceso a la información.

Anteriormente, Bonito y dos Santos (2018) hablaron de Accesibilidad Comunicativa como un concepto que "debe ser comprendido desde un ámbito social que engloba las cuestiones culturales hasta el ámbito político que configura las legislativas" (p. 208), resaltando que todas las culturas comunicativas hegemónicas vigentes fueron desarrolladas y creadas a partir de las lógicas de personas que ven y oyen sin dificultades. Sobre esta base, se pretende ampliar la dimensión de accesibilidad comunicacional llegando a intervenir los componentes de participación del diseño universal en dos sentidos: primero, la participación como un papel activo y significativo en la toma de decisiones que afectan los intereses propios, y segundo, la participación como un proceso comunitario en el que un todo (población/comunidad con discapacidad) determina su condición.

En síntesis, se requiere analizar con mayor profundidad lo que significa eliminar barreras de información y comunicación para las personas con discapacidad, no solo en la comunicación interpersonal, en la comunicación escrita y en la comunicación digital, sino también en otros escenarios comunicativos. Como proponen Cuesta y Meléndez (2016) en una línea de trabajo para la inclusión de las PCD a la ciudad: garantizando la participación, abordando el proceso comunicativo no solo desde su condición instrumental sino especialmente como "un proceso donde se ponen en común sentidos y significados a partir de un proceso en donde los sujetos simulan los objetos mentales del otro" (p. 7). Más claramente, se trata de

la participación de estas personas en el espacio y la posibilidad de compartir con las otras personas que configuran dicho espacio. Tal participación es intrínsecamente un hecho comunicativo, dado que se realiza mediante intercambios de orden simbólico que procuran la construcción de un sentido común: lo público. (Cuesta \& Meléndez-Labrador, 2019, p. 278)

Teniendo clara esta ampliación del concepto, ahora pasamos a identificar una estrategia de uso y visibilización de la ss que podría contribuir a su preservación como elemento de la Accesibilidad Comunicativa Universal. Se encontró que las guías y manuales de comunicación inclusiva son herramientas de fortalecimiento de los procesos de producción de contenidos sobre las personas con discapacidad, y que también pueden orientar estrategias de comunicación que intervienen en dinámicas de relacionamiento de este grupo minoritario.

En este sentido, las guías y manuales sobre comunicación y discapacidad desarrolladas por entidades públicas, privadas, expertos y organizaciones no gubernamentales permiten trascender de la teoría en espacios académicos a la práctica (Francisco, 2003), convirtiéndose en dispositivos para revertir el impacto de la discriminación y la exclusión anquilosadas en la realidad de las PCD, al tiempo que, entre otras, visibilizan la Ls. En otras palabras, tanto medios de comunicación como periodistas, comunicadores, estudiantes de estas áreas y demás personas que intervienen en procesos de comunicación y discapacidad tienen un papel fundamental como agentes de cambio, no solo en la formación de la imagen social de las personas con discapacidad, sino también en la eliminación de barreras de participación y de acceso a la información, incluyendo el uso y preservación de la lengua de señas como forma de comunicación de una población minoritaria con discapacidad auditiva. 


\section{DISERTACIONES}

\section{ESTUDIOS}

Grupos minoritarios y estigmatizados: diversidad funcional, religiosa, étnica, afectivo-sexual o de identidad de género en la comunicación

ISSN: $1856-9536$

Doi: https://doi.org/10.12804/revistas.urosario.edu.co/disertaciones/a.10127

Volumen 15, Número 1 / Enero-junio 2022

Versión PDF para imprimir desde

http://revistas.urosario.edu.co/index.php/disertaciones

Con todo lo anterior es preciso identificar, más allá de los aspectos legales e históricos, cuál es el lugar que ocupa la ss en la Accesibilidad Comunicativa Universal que promulgan las guías y manuales de comunicación y discapacidad, con el propósito de comprender la manera como este material y los procesos de capacitación que apoya, pueden contribuir al reconocimiento y promoción de lengua de señas (como lo exige la Convención) y, a su preservación, como es requerido por toda lengua minoritaria.

\section{Metodología}

Se realizó una investigación documental en la que se revisaron 22 guías y manuales de comunicación y discapacidad en español, recopilados y presentados por la autora a través del Observatorio Latinoamericano de Discapacidad y Comunicación (Obladic), en su página web. Se accedió al listado en el mes de diciembre del 2020 en el enlace www.obladic.org/guias (véase tabla 1). Se utilizó el análisis de texto para identificar tanto de manera general como en detalle - lo que implicó un conocimiento previo del tema a analizar- las pautas y recomendaciones concernientes a las formas de comunicación de y con las PS. Así, desde un enfoque hermenéutico, se buscaron y clasificaron palabras y frases características de los textos, de manera comparativa entre las guías, hasta identificar los valores o significados latentes (Larsen, 2002) de Accesibilidad Comunicativa Universal de estas publicaciones y del lugar que ocupa la ts en ese modelo.

Tabla 1. Guías y manuales sobre comunicación y discapacidad analizados

\begin{tabular}{|c|c|c|c|}
\hline Año & Nombre de la publicación & Autor & País \\
\hline 2001 & $\begin{array}{l}\text { Guía de estilo periodístico para informar sobre } \\
\text { discapacidad. Gobierno de Colombia. }\end{array}$ & $\begin{array}{l}\text { Óscar Cortés y Maria Stella } \\
\text { Zorro }\end{array}$ & Colombia \\
\hline 2002 & $\begin{array}{l}\text { Cómo mejorar la comunicación social sobre } \\
\text { discapacidad. Guía práctica para entidades. }\end{array}$ & $\begin{array}{l}\text { Antonio Sánchez de Amo y } \\
\text { Susana Márquez }\end{array}$ & España \\
\hline 2003 & $\begin{array}{l}\text { Medios de Comunicación y Discapacidad. Un análisis de } \\
\text { la cobertura periodística sobre inclusión social. }\end{array}$ & Francisco Severino (Ed.) & Brasil \\
\hline 2004 & Pautas de estilo periodístico sobre discapacidad. & Alejandra Noseda & Argentina \\
\hline 2004 & $\begin{array}{l}\text { Guía para autogestores. Hacer que tu historia se } \\
\text { escuche. }\end{array}$ & Real Live Media & Portugal \\
\hline 2005 & $\begin{array}{l}\text { Comunicación para todos. Pautas para la comunicación } \\
\text { accesible. }\end{array}$ & Grupo Telefónica & España \\
\hline 2006 & $\begin{array}{l}\text { Guía de estilo sobre discapacidad para profesionales de } \\
\text { los medios de comunicación. }\end{array}$ & José Luis Fernández & España \\
\hline 2010 & $\begin{array}{l}\text { Por una comunicación inclusiva sobre discapacidad. } \\
\text { Manual para comunicadores. }\end{array}$ & CONDIS & Bolivia \\
\hline 2010 & $\begin{array}{l}\text { Medios de comunicación y discapacidad. Un aporte para } \\
\text { la cobertura periodística sobre inclusión social. }\end{array}$ & Agencia global de Noticias & Paraguay \\
\hline 2011 & Guía para una comunicación incluyente. & CIESPAL & Ecuador \\
\hline
\end{tabular}




\section{DISERTACIONES}

ESTUDIOS

Grupos minoritarios y estigmatizados: diversidad funcional, religiosa, étnica, afectivo-sexual o de identidad de

\begin{tabular}{|c|c|c|c|}
\hline Año & Nombre de la publicación & Autor & País \\
\hline 2011 & $\begin{array}{l}\text { Diferentes: guía ilustrada sobre la diversidad y la } \\
\text { discapacidad. }\end{array}$ & Angels Ponce & España \\
\hline 2013 & $\begin{array}{l}\text { Manual Buenas Prácticas en la Interacción con las } \\
\text { personas con Discapacidad. }\end{array}$ & Cormin & España \\
\hline 2013 & $\begin{array}{l}\text { Guía de buenas prácticas sobre personas con } \\
\text { discapacidad para profesionales de la comunicación: } \\
\text { manual de estilo. }\end{array}$ & Rosa Álvarez & España \\
\hline 2013 & $\begin{array}{l}\text { Manual sobre comunicación y discapacidad: el } \\
\text { compromiso desde la información. }\end{array}$ & $\begin{array}{l}\text { Universidad Católica } \\
\text { Boliviana "San Pablo" }\end{array}$ & Bolivia \\
\hline 2014 & $\begin{array}{l}\text { Comunicación que no discrimina. Guía para } \\
\text { comunicadores. }\end{array}$ & $\begin{array}{l}\text { Ministerio de Cultura de } \\
\text { Perú }\end{array}$ & Perú \\
\hline 2015 & $\begin{array}{l}\text { Comunicación para la inclusión. Manual de estilo para el } \\
\text { abordaje periodístico de la discapacidad en Venezuela. }\end{array}$ & Indira Ramírez & Venezuela \\
\hline 2016 & $\begin{array}{l}\text { Criterios comunicativos para la inclusión de personas en } \\
\text { condición de discapacidad a las dinámicas urbanas. }\end{array}$ & $\begin{array}{l}\text { Óscar Cuesta y Sandra } \\
\text { Meléndez }\end{array}$ & Colombia \\
\hline 2018 & Accesibilidad web. WCAG 2.1 de manera sencilla. & Olga Revilla y Olga Carreras & España \\
\hline 2018 & $\begin{array}{l}\text { Comunicación y discapacidad. Guía de buenas prácticas } \\
\text { para trabajadoras y trabajadores de la comunicación. }\end{array}$ & Mides & Uruguay \\
\hline 2020 & Manual de comunicación y discapacidad. & Almudena Rascón & Chile \\
\hline s.f. & $\begin{array}{l}\text { Guía de orientaciones para la atención de personas con } \\
\text { discapacidad en servicios públicos, privados y de interés } \\
\text { social. }\end{array}$ & $\begin{array}{l}\text { Universidad Católica } \\
\text { Boliviana "San Pablo" }\end{array}$ & Bolivia \\
\hline s.f. & Medios y discapacidad. & Verónica González Bonet & Argentina \\
\hline
\end{tabular}

\section{Fuente: elaboración propia.}

\section{Resultados}

En general, con el análisis textual se encontró información sobre lenguaje, recomendaciones para la interacción, acceso a la información, a los medios de comunicación y pautas para el ejercicio profesional e inclusivo del periodismo. En detalle, las guías abordaron la discapacidad a partir de estas áreas: definición y modelos conceptuales; contexto cultural; clasificación médica; barreras del entorno; aspectos históricos y legales; lenguaje no discriminatorio; y pautas de accesibilidad comunicacional.

Con el fin de cumplir con el propósito de este estudio, se agruparon los datos relacionados con las PS/PCDA en las categorías (a) contextualización interseccional, que explica la realidad social de las personas con discapacidad desde la interacción de factores sociales, económicos, políticos, culturales y simbólicos; (b) lenguaje, que enumera los términos y expresiones que no discriminan y que son considerados como correctos para el tratamiento de información sobre PS/PCDA; (c) interacción, que detalla los aspectos básicos a tener en cuenta para establecer 


\section{DISERTACIONES}

ESTUDIOS

contacto interpersonal con una PS/PCDA; (d) formas y medios de expresión, que detalla las formas de expresión y los medios de apoyo para la comunicación con personas que tienen limitación auditiva; y (e) participación, en la cual se presentan tácticas en las que se involucra a la PS/PCDA en toma de decisiones y definición de situaciones de las que hace parte. A continuación, se exponen los hallazgos en la tabla 2.

\section{Tabla 2. Características de la lengua de señas en la comunicación inclusiva}

Contextualización interseccional

» Tenga en cuenta y mencione la realidad social de las personas con discapacidad desde un enfoque interseccional.

» Mencione las barreras educativas para la alfabetización en ss o del español como segundo idioma, no hable de problemas de aprendizaje de las ps.

» Mencione el valor de la ss como forma de comunicación oficial de las ps en el marco de los derechos lingüísticos.

Lenguaje

» Expresión incorrecta: sordo, mudo, sordomudo, sordito, hipoacúsico, lenguaje de señas.

» Expresión correcta: persona sorda, persona sordociega, persona con discapacidad auditiva, persona con sordera, persona con hipoacusia, persona con limitación auditiva, lengua de señas.

Interacción

» General:

- Dé la mano en señal de saludo.

- Sitúese a su altura, sobre todo si se trata de niños.

- Diríjase a la Ps/PCDA cuando le esté mirando, evite hacerlo si se encuentra de espaldas.

- Llame la atención de la Ps/PCDA dando un ligero toque en su hombro o brazo, golpee suavemente el suelo o la mesa para que sienta las vibraciones o haga una discreta seña antes de iniciar la conversación estando dentro de su campo visual (evite tocar a la persona estando de espaldas).

- Avise a la Ps/PCDA cuando quiera hablar con ella, indicándole el tema de conversación y, cada vez que varíe, hágaselo saber.

- Mientras caminen, es preferible no conversar.

- Brinde información complementaria sobre cualquier estímulo auditivo (música de fondo, mensajes por megáfono, instrucciones verbales a otras personas). Eso le permite a la PS/PCDA entender mejor las reacciones de otras personas y adecuar su comportamiento de un modo más eficaz.

- Proporcione alternativas a la comunicación oral como apoyos visuales, lenguaje corporal, gestos naturales y signos sencillos, sin sobreactuar. Los movimientos faciales y corporales ayudarán a la Ps/PCDA a comprender, especialmente si los interlocutores tienen barba o bigote.

- Asegúrese de que el lugar donde interactúan esté bien iluminado, de ser posible, con buena luz en el rostro y ubicados a menos de 3 metros entre sí.

- No asuma que la persona con pérdida auditiva también tiene una discapacidad cognoscitiva, como retraso mental.

»Periodística:

- Es importante consultar a la Ps/PCDA si usa fundamentalmente ss, lectura labial, lenguaje escrito o lenguaje gestual y, las condiciones más apropiadas para una entrevista (por ejemplo, un ambiente muy ruidoso no favorece la escucha y la comprensión de las preguntas). Si la PS/PCDA está acompañada por otra persona, el camarógrafo debe enfocarla a ella y no a su acompañante.

- El lenguaje debe ser conciso y claro durante una entrevista. Se deben evitar tecnicismos y anglicismos.

- En una entrevista grupal organice el diálogo de manera que no hablen al mismo tiempo, dando espacio para que la Ps/PCDA se ubique y sepa quién le está hablando.

- Evite interponerse entre la Ps/PCDA y su(s) interlocutor(es) mientras realiza una entrevista o toma fotografías. 


\section{DISERTACIONES}

Grupos minoritarios y estigmatizados: diversidad funcional, religiosa, étnica, afectivo-sexual o de identidad de

Formas y medios de expresión 1: lectura labio facial

»Colóquese siempre delante de la Ps/PCDA para facilitar la lectura de los labios y que pueda haber un contacto visual y facial adecuado. Evite moverse.

» Vocalice correctamente, pero sin exagerar los gestos sobre lo que está explicando. Tampoco eleve el volumen de la voz.

"Utilice palabras sencillas y fáciles de leer en los labios.

» Articule las palabras en forma clara y pausada, siempre con un tono natural, evitando hablar deprisa o demasiado despacio.

» No mastique chicle.

»Evite taparse la boca, poner las manos delante de esta o voltearse cuando está interactuando con una PS/PCDA.

Formas y medios de expresión 2: lengua de señas

» Tenga en cuenta que la videollamada constituye un medio de apoyo importante para la comunicación de la Ps/PCDA, ya que les permite ver al interlocutor y comunicarse en directo a través de la ss o la lectura labio facial.

» Use intérprete de ss únicamente para aquellas personas que han recibido formación avanzada.

» Diríjase a la Ps/PCDA cuando se habla, y no a su intérprete.

»El intérprete debe conservar el principio de confidencialidad y respetar la forma de pensar y expresarse de la PS/PCDA, especialmente en la gesticulación.

» La ss también es un recurso utilizado para comunicación a través de medios audiovisuales como televisión, cine e Internet. En estos se debe ofrecer información básica sobre la organización del sitio, datos de contacto y la manera de acceder a los contenidos.

» En servicios de salud y unidades de emergencia, instituciones educativas, lugares de espectáculo y ocio, lugares de trabajo, etc., debe disponerse de un intérprete de señas permanente.

» Accesibilidad web:

- El audio debe estar sincronizado con la lengua de señas.

- También puede ofrecer un diccionario de lengua de señas.

» Pautas periodísticas:

- Los camarógrafos no deben enfocar al acompañante sino a la persona que da la información (PS/PCDA). Siga las anteriores recomendaciones con respecto a la interpretación en LS.

- Al citar o parafrasear a una persona usuaria de Ls, lo apropiado es indicar en la primera referencia que las respuestas se expresaron a través de esta lengua.

- El intérprete de ss debe aparecer con un fondo blanco y ubicarse correctamente en el encuadre. 


\section{DISERTACIONES}

ESTUDIOS

Formas y medios de expresión 3: lenguaje escrito

» También puede valerse del mensaje escrito, ya sea manual o por teletexto como el chat o la mensajería electrónica.

» Sea breve y claro, tenga en cuenta que una PS/PCDA le puede responder con frases cortas y concretas, y que no todas saben leer o escribir.

"En casos de sordera prelocutiva, ${ }^{7}$ es posible que la PS/PCDA posea un vocabulario relativamente restringido, encontrando dificultades para entender términos poco usuales o textos largos y complejos.

» Los medios audiovisuales como televisión, cine e Internet se valen de subtítulos para reproducir en textos escritos los mensajes hablados y los sonidos asociados a las imágenes que proyectan como sucesos (sonido ambiente) o entonación (gritos).

» La subtitulación aparece generalmente en la parte inferior de la pantalla y permite a las PS/PCDA acceder a la información suministrada.

» Accesibilidad web:

- En Internet las PS/PCDA pueden no percibir los avisos sonoros ni acceder a la banda de audio de los elementos multimedia, por lo que el audio también debe estar sincronizado con los subtítulos de forma abierta o cerrada con tecnología SMIL u otro similar que soporte el reproductor.

- Se debe ofrecer información básica escrita de manera clara y sencilla sobre la organización del sitio, datos de contacto y el mapa de navegación.

- Se requiere etiquetar la alternativa textual para un texto sincronizado y para la información contenida en imágenes y diagramas.

- Ofrecer enlaces a descripciones del contenido de audio o subtítulos.

»Pautas periodísticas:

- Resalte mediante subrayado o negrita las palabras claves para apoyar la información.

- Evite frases subordinadas y que contengan muchos enlaces.

- Las emisoras de radio deberían ofrecer páginas web accesibles con textos de fácil comprensión de los distintos programas y acompañados de imágenes.

- Se puede informar a través de otros medios escritos o que incluyan interpretación en ts que, en el caso de personas con hipoacusia (pérdida auditiva parcial) o con resto auditivo, pueden acceder a la radio: (a) usando audífonos que amplifican el sonido; (b) elevando el volumen del transistor; y (c) usando auriculares para acercar la señal auditiva.

Formas y medios de expresión 4: otros apoyos visuales y sonoros

» Generar, adquirir o acondicionar apoyos técnicos con estas adaptaciones acústicas:

- Bucles magnéticos o lazos de inducción magnética: sistemas reductores del ruido que mejoran la audición de las PS/ PCDA que utilizan audífonos o tienen implantes cocleares. Se instalan en mostradores de atención al público, salas de conferencias, auditorios, cines, teatros, instalaciones de transporte, etcétera.

- Sistema de fm: las emisoras o equipos de frecuencia modulada amplifican la voz de la persona que habla por encima del ruido ambiente y la hacen llegar directamente a las prótesis de las PS/PCDA que utilizan audífonos o tienen implantes cocleares. Constan de dos componentes: un emisor y un receptor que se conecta al audífono o al implante. Se utilizan fundamentalmente como ayuda técnica de uso individual en procesos de enseñanza.

» Disponer de ayudas visuales en el espacio físico para alarmas y demás información de emergencia.

» Utilizar elementos gráficos como una oreja o unas manos en posición de movimiento de los dedos, para advertir que se dispone de subtitulación o interpretación de Ls.

7 Se refiere a personas con sordera previa a la adquisición del lenguaje, por ejemplo, niños y personas cuya mudez es consecuencia de una sordera prelocutiva. 


\section{DISERTACIONES}

Grupos minoritarios y estigmatizados: diversidad funcional, religiosa, étnica, afectivo-sexual o de identidad de

Participación

» Generalidades:

- Indague cuál es la forma de comunicación que requiere la PS/PCDA: lectura de labios, lengua de señas, escritura, etcétera.

- Si va a interactuar con una persona con hipoacusia pregúntele cuál sería la mejor ubicación.

- Si hay varias personas en una reunión ubíquelas en semicírculo para que la PS/PCDA tenga una visión más amplia y sepa quién está hablando.

» Lengua de señas:

- Permita que la PS/PCDA asista a la entrevista con su intérprete de ls y defina el lugar donde estará su intérprete.

- Dé el tiempo necesario para que el intérprete de ss haga su trabajo.

- No se limite a contratar un intérprete de lengua de señas, ya que cuando esta falta las PS/PCDA que requieren el acompañamiento no podrán comunicarse con las personas oyentes.

Fuente: elaboración propia.

A continuación, se amplían los aspectos más destacados del estudio:

\section{Contextualización interseccional}

Algunas guías o manuales le otorgaron espacio a información sobre la realidad social de las PCD de manera muy ilustrativa, por ejemplo, la guía del Ministerio de Cultura de Perú (2014) hace énfasis en el enfoque interseccional para comprender la realidad social de la población con discapacidad así:

Resulta fundamental la utilización de un enfoque interseccional para el análisis y real comprensión de los niveles de discriminación. Dicho enfoque toma en consideración el contexto histórico, social y político, y reconoce la experiencia genuina del individuo basada en la intersección de todos los factores relevantes. (p. 22)

En esta línea, la guía de Francisco (2003) hace una distinción entre la inclusión y la integración, usando como ejemplo la Lengua de Señas Brasilera (Libras). Critica que un modelo de integración "incentiva a las personas con discapacidad a seguir modelos, no valorizando, por ejemplo, otras formas de comunicación como la Libras. Seríamos entonces un bloque mayoritario y homogéneo de personas sin discapacidad, rodeada por las que presentan diferencias" (p. 21). El autor añade que el empoderamiento y la plena participación se da en una sociedad inclusiva "en la cual cada uno de nosotros y todos nosotros seamos considerados parte integral del todo, de la comunidad que, a su vez, es responsabilidad del conjunto de sus miembros" (p. 31).

Otro ejemplo es el de la guía de Ciespal (2011) cuando advierte: "Tampoco es igual la situación de una persona sorda con oportunidades educativas desde su infancia, frente a una persona sorda sin ningún apoyo educativo" (p. 40). 


\section{DISERTACIONES}

ESTUDIOS

Grupos minoritarios y estigmatizados: diversidad funcional, religiosa, étnica, afectivo-sexual o de identidad de

género en la comunicación

ISSN: $1856-9536$

Doi: https://doi.org/10.12804/revistas.urosario.edu.co/disertaciones/a.10127

Volumen 15, Número 1 / Enero-junio 2022

Versión PDF para imprimir desde

http://revistas.urosario.edu.co/index.php/disertaciones

\section{Lenguaje}

De manera general se encontró que todas las guías ofrecen información sobre el lenguaje inclusivo y la terminología correcta para referirse y representar a las PCD en los medios. Por ejemplo, el texto de Francisco (2003) expone que (a) la comunicación de señas de y entre ps constituye una lengua y no un lenguaje; (b) la comunicación mediante gestos, que incluye o no a ps constituye un lenguaje gestual; (c) otra aplicación del concepto lenguaje se refiere a las posturas y actitudes humanas que comunican de forma no verbal, es decir, el lenguaje corporal.

\section{Formas y medios de expresión}

De manera poco detallada la guía de Álvarez (2013) advierte: "Las personas con discapacidad auditiva utilizan medios de apoyo a la lengua oral o bien la lengua de signos, por lo que no siempre hay que deducir que todas se comunican en lengua de signos" (p. 20).

En contraste, la completa publicación de Francisco (2003) también pone en contexto la definición de persona sorda, aclarando que no presentan, necesariamente, un problema de voz. Explica que no hablan porque no escuchan, teniendo así mucha dificultad de expresarse a través del portugués, lo que los lleva a optar casi siempre por la Libras. Resalta además que "la Ley Federal N. ${ }^{\circ} 10436$ del 24 de abril del 2002, reconoce a la Libras y otros recursos de expresión a ella asociados como medio legal de comunicación" (p. 42).

Siguiendo el tema, las guías de Ramírez (2015) y Mides (2018) también abordan la discapacidad desde una perspectiva histórica y legal. Ramírez exalta el reconocimiento oficial que Venezuela da en la Constitución Nacional de 1999 a la Lengua de Señas Venezolana (LSV), como medio de expresión y comunicación de las PS/PCDA. Más adelante recuerda que en la Constitución "se le indica a los medios su empleo para garantizar el derecho a las personas con deficiencias de audición a ser informado, en su lengua, a través de la televisión pública y privada” (2015, p. 14). Por su parte, Mides presenta la Ley 17.378 de 2001 en la que se reconoce la Lengua de Señas Uruguaya (LSU) como lengua oficial de ese país. Agrega que

todos los individuos tenemos la facultad del lenguaje y el derecho a adquirir una lengua. En la singularidad de las personas sordas su lengua natural es la lengua de señas. Esta lengua tiene su especificidad tal como las demás lenguas desarrolladas por diferentes sociedades y culturas, por lo cual es necesario el aprendizaje para la adquisición y el desarrollo de las destrezas necesarias que permitan hablar la lengua con fluidez. Procurar que niñas y niños sordos y sordas accedan a la educación formal desde el ciclo preescolar, y adquieran la ısu con la calidad debida, es esencial en tanto la alfabetización es un pilar básico para toda sociedad que se desee inclusiva. (2018, p. 13)

Con respecto a los asuntos estructurales de la ıs, Rascón (2020) explica: "Hay que considerar que la Lengua de Señas tiene una estructura gramatical propia, distinta a la del español, y no es una codificación del español como a veces se cree" (p. 2) y elimina el prejuicio de que solo existe una ts común a todas las personas sordas del mundo: "En cada país existe una Lengua de Señas propia, que además varía incluso a nivel regional" (p. 34). En su caso, Ramírez (2015) aclara brevemente que "la Lsv tiene una gramática y un vocabulario propios, que la diferencian de las lenguas de señas de otros países" (p. 29).

Mides (2018) explica también que la interpretación en ısu "no es ni puede ser textual, sino que es una representación conceptual del contenido del mensaje, del diálogo que se está estableciendo” (p. 20) y aclara: 


\section{DISERTACIONES}

ESTUDIOS

Grupos minoritarios y estigmatizados: diversidad funcional, religiosa, étnica, afectivo-sexual o de identidad de

género en la comunicación

ISSN: 1856-9536

Doi: https://doi.org/10.12804/revistas.urosario.edu.co/disertaciones/a.10127

Volumen 15, Número 1 / Enero-junio 2022

Versión PDF para imprimir desde

http://revistas.urosario.edu.co/index.php/disertaciones

Existe la creencia errónea de que las personas en situación de discapacidad auditiva pueden escribir y leer con facilidad. Esto no es así para todos los casos. El español escrito para las personas sordas es su segunda lengua. No tiene que ver con su capacidad de aprendizaje, sino con el manejo de una segunda lengua. La Lsu es la primera lengua de una persona sorda y así lo reconoce la legislación de nuestro país. (2018, p. 21)

Desde otra perspectiva, en la extensa obra de Revilla y Carreras (2018) se abordan problemas comunes de accesibilidad que crean situaciones discapacitantes en relación con las PS/PCDA así: "Los problemas que experimenta una persona sorda para entender un vídeo en Internet son los mismos problemas que experimenta un oyente con el mismo vídeo en un entorno ruidoso" (p. 12).

Para cerrar este apartado, es necesario señalar que en las guías y manuales analizados no se pone en contexto la posibilidad de que las PS/PCDA puedan utilizar una LS casera y que también generan en cada lugar en el que estas personas puedan establecerse como comunidad, por ejemplo, en un sitio de trabajo (Meléndez-Labrador, 2016). Tampoco presentan información sobre asuntos que competen a las personas sordociegas (PSC) en relación con la lengua de señas táctil (LST) o lengua de señas adaptada al tacto y el sistema Tadoma (Condis, 2010), más allá de la definición de los términos dactilografía y dactilología (Fernández, 2006; Saldarriaga, 2014). Fernández (2006) describe someramente que en la LST se colocan las manos de la psc sobre las de quien le habla para poder percibir el mensaje, y añade: "Las manos de la persona sordociega se colocan en el ángulo formado entre el pulgar y el índice de su interlocutor para seguir el mensaje” (p. 134). Por su parte, Condis (2010) explica que el sistema Tadoma consiste en poner dos dedos sobre los labios y otro en la garganta, para percibir el movimiento de labios y la vibración de las cuerdas vocales. Este aspecto es muy importante teniendo en cuenta que, en la Ley 27 de 2007 de España "se determina el reconocimiento y regulación de la LSE como lengua propia de las personas sordas y sordociegas" (p. 36).

\section{Participación}

Werneck (2005) presenta a las personas con discapacidad como protagonistas de las historias - deportistas paralímpicos que se volvieron héroes-y como fuentes de información económica o literaria. Asimismo, llama la atención con interrogantes como ¿De qué manera las personas que utilizan lengua de señas o practican lectura labial pueden participar en procesos de construcción de política pública o reuniones comunitarias?

Nuevamente, la guía de Francisco presenta una distinción a la Libras (lengua de señas brasilera), reconociendo la intensa lucha que el movimiento de personas con sordera ha desarrollado en el país, afirmando que Libras es un término con el cual se identifica la comunidad brasilera de personas con sordera, y continúa:

El término se inserta en la tradición y es extremadamente querido por la mencionada comunidad. Mantener el término indica el profundo respeto del pueblo brasilero hacia esta comunidad que se desea ayudar y promover, tanto por razones humanitarias como para fomentar la conciencia social y la ciudadanía. Mientras tanto, en el registro lingüístico internacional los idiomas naturales de todos los pueblos del planeta reciben una sigla de tres letras como por ejemplo AsL (American Sign Language). Por lo tanto será necesario crear otra sigla. Parece que esta preocupación aún se encuentra alejada de la esfera brasilera. (2016, p. 163) 


\section{DISERTACIONES}

\section{ESTUDIOS}

Adicionalmente, sobre el papel de la Libras en los medios de comunicación la guía de Francisco (2003) explica que existen personas con sordera oralizadas, que pueden comprender más fácilmente el segundo idioma hablado y escrito (en este caso el portugués) y el grupo de personas no oralizadas que

defienden que la tecla "closed caption" no debe sustituir la presencia de un traductor de Libras en la pantalla del televisor, porque la mayoría de personas con sordera en el Brasil no entiende el portugués escrito, por lo menos en la velocidad de los subtítulos. (p. 113)

En el caso boliviano, la Universidad Católica Boliviana "San Pablo" (2013), expone que el derecho a la comunicación e información de su país "abarca las libertades del derecho a la información (expresión, búsqueda y recepción), e incorpora conceptos nuevos como accesibilidad, participación, expresión desde la diversidad cultural, y con un destino de construcción de ciudadanía” (p. 13), aunque no se detalla de qué manera en la práctica comunicativa se puede desarrollar el componente participativo.

Para cerrar, como se evidenció hasta ahora, la guía de Francisco (2003) es la publicación que más responde a un modelo de Accesibilidad Comunicativa Universal, aun así, se considera que se puede desarrollar más la perspectiva participativa en el diseño de este tipo de guías sobre comunicación y discapacidad. Por ejemplo, en este manual se usa un caso para ilustrar el diseño universal así:

Una empresa que contrata a un intérprete de Libras para los empleados sordos, pero no prepara un ambiente profesional para la inclusión efectiva. El día en que el intérprete falta, los otros empleados no pueden comunicarse con las personas que tienen sordera. Aunque la decisión de la empresa haya sido correcta, en ella se nota una ausencia de reflexión más profunda sobre la diversidad, lo que reduce su alcance al límite de la providencia puntual, descontextualizada de una verdadera perspectiva de inclusión. (p. 26)

Sin embargo, es de resaltar que la empresa puede disponer de tiempo y recursos para que las ps enseñen la Ls a su jefe y compañeros con quienes interactúa de manera constante, como se evidenció en el estudio de Meléndez-Labrador (2016). De hecho, puede abrir espacios de visibilización y promoción de la Ls a partir del involucramiento de las ps en los procesos de comunicación interna y externa de la empresa, promoviendo el empoderamiento y la autonomía en los procesos de inclusión laboral.

\section{Conclusiones}

Basados en el modelo de Accesibilidad Comunicativa Universal establecido en este estudio, y que contempla cinco dimensiones: (a) contextualización interseccional, (b) lenguaje, (c) interacción, (d) formas y medios de expresión, y (e) participación (véase figura 1), se pudo observar que el lugar que ocupa la ss hasta ahora en las guías de comunicación y discapacidad publicadas en español es, por un lado, una forma de expresión en soportes y formatos alternativos que garantizan la información accesible y la accesibilidad a la información, y por el otro, un elemento clave del componente participativo. 


\section{DISERTACIONES}

Grupos minoritarios y estigmatizados: diversidad funcional, religiosa, étnica, afectivo-sexual o de identidad de género en la comunicación

ISSN: $1856-9536$

Doi: https://doi.org/10.12804/revistas.urosario.edu.co/disertaciones/a.10127

Volumen 15, Número 1 / Enero-junio 2022

Versión PDF para imprimir desde

http://revistas.urosario.edu.co/index.php/disertaciones

Sin embargo, llamó la atención la falta de reconocimiento de la ts como lengua minoritaria oficial en cada territorio y, el escaso abordaje de las implicaciones de este modo de comunicación con respecto a la comunidad que la práctica. Esto incluye reconocer el papel de la us en la garantía de los derechos de las personas con discapacidad y el papel de una Accesibilidad Comunicativa Universal, de comunicadores y medios, en la supervivencia y expansión de la Ls, así como en la inclusión social y ciudadana de esta población.

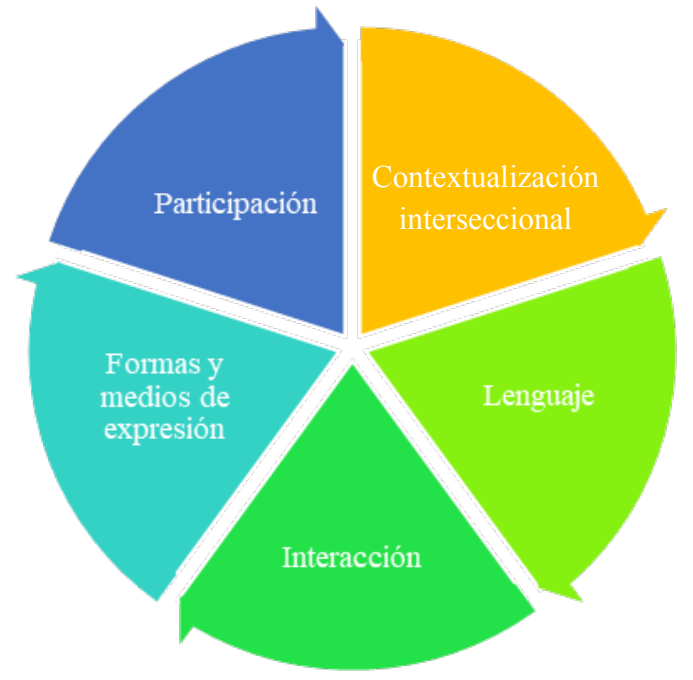

Figura 1. Modelo de la Accesibilidad Comunicativa Universal

Fuente: elaboración propia.

En ese orden, las guías que se desarrollen dentro del modelo de Accesibilidad Comunicativa Universal servirán no solo para orientar la producción de contenidos sobre discapacidad, sino también para diseñar procesos de co-construcción de significado. Esto exige tomar en cuenta aspectos de la comunicación participativa y dialógica que involucre asuntos de participación política, movilización y las condiciones para que la comunicación sea realizada por, con y para la comunidad (Peruzzo, 2020) para el caso, con diversidad funcional. No se trata solo de narrativas accesibles sino de procesos comunicativos accesibles para todos, universales, que vayan desde la eliminación de barreras de acceso a la información, hasta la implementación de estrategias comunicativas que puedan suscitar la autoinclusión social y ciudadana de esta población favoreciendo su autonomía. 


\section{DISERTACIONES}

ESTUDIOS

Grupos minoritarios y estigmatizados: diversidad funcional, religiosa, étnica, afectivo-sexual o de identidad de

género en la comunicación

ISSN: $1856-9536$

Doi: https://doi.org/10.12804/revistas.urosario.edu.co/disertaciones/a.10127

Volumen 15, Número 1 / Enero-junio 2022

Versión PDF para imprimir desde

http://revistas.urosario.edu.co/index.php/disertaciones

\section{Referencias}

1. Adam, R. (2015). Dissemination and transfer of knowledge to the Deaf community. En E. Orfanidou, B. Woll \& G. Morgan (Eds.), Research Methods in Sign Language Studies: A Practical Guide, 41-52. John Wiley \& Sons. https://doi.org/10.1002/9781118346013

2. Agencia global de noticias. (2010). Medios de comunicación y discapacidad. Un aporte para la cobertura periodística sobre inclusión social. Agencia de Noticias; Infancia de la Asociación Global; Andi América Latina. https://sid.usal.es/idocs/F8/FDO24909/medios-discapacidad.pdf

3. Ahmad, M. (2008). What factors contribute to sign language endangerment? [MA dissertation]. School of Oriental and African Studies.

4. Álvarez, R. (2013). Guía de buenas prácticas sobre personas con discapacidad para profesionales de la comunicación: manual de estilo. Consejería de Salud y Bienestar Social. https://periodistasandalucia.es/ wp-content/uploads/2015/05/GUIA-DE-BUENAS-PRACTICAS-CSBS-25-JULIO-2013-1.pdf

5. Austin, P. K., \& Sallabank, J. (Eds.). (2011). The Cambridge handbook of endangered languages. Cambridge University Press.

6. Bickford, J., Lewis, M., \& Simons, G. (2015). Rating the vitality of sign languages. Journal of Multilingual and Multicultural Development, 36(5), 513-527. https://doi.org/10.1080/01434632.2014.966827

7. Bonito, M., \& Dos Santos, L. C. (2018). Procesos comunicacionales inclusivos: una mirada bajo la óptica de la accesibilidad comunicativa. Revista Latinoamericana de Ciencias de la Comunicación, 15(29). http:// revista.pubalaic.org/index.php/alaic/article/view/1321/576

8. CIESPAL. (2011). Guía para una comunicación incluyente. Centro Internacional de Estudios Superiores para América Latina; Vicepresidencia de la República de Ecuador. http://www.larediberoamericana.com/ wp-content/uploads/2012/07/Guia-para-una-comunicacion-incluyente.pdf

9. Condis. (2010). Por una Comunicación inclusiva sobre discapacidad, Manual para Comunicadores. Oruro.

10. Cormin. (2013). Manual buenas prácticas en la interacción con las personas con discapacidad. Gobierno de Navarra. http://issuu.com/anundis/docs/buenas_pr_cticas_en_la_interacci__ee=6720722/4693251

11. Cortés, O., \& Zorro, M. S. (2001). Guía de estilo periodístico para informar sobre discapacidad. Gobierno de Colombia; Ministerio de Educación de Colombia. https://silo.tips/download/guia-de-estilo-periodisticopara-informar-sobre-discapacidad

12. Cuesta, O., \& Meléndez, S. (2016). Criterios comunicativos para la inclusión de personas en condición de discapacidad a las dinámicas urbanas. Fundación Universitaria Los Libertadores. https://www.academia. edu/31668139/Criterios_comunicativos_para_la_inclusi\%C3\%B3n_de_personas_en_condici\%C3\%B3n_ de_discapacidad_a_las_din\%C3\%A1micas_urbanas?auto_accept_coauthor=true

13. Cuesta, Ó., \& Meléndez-Labrador, S. (2019). Discapacidad, ciudad e inclusión cultural: consideraciones desde la comunicación urbana. Revista EURE, Revista de Estudios Urbano Regionales, 45(135), 273-282. http://dx.doi.org/10.4067/S0250-71612019000200273

14. Esteban, M. L., \& Ramallo, F. (2019). Derechos lingüísticos y comunidad sorda: claves para entender la minorización. REVLES, (1), 20-52. https://revles.es/index.php/revles/article/view/19 


\section{DISERTACIONES}

ESTUDIOS

Grupos minoritarios y estigmatizados: diversidad funcional, religiosa, étnica, afectivo-sexual o de identidad de

género en la comunicación

ISSN: $1856-9536$

Doi: https://doi.org/10.12804/revistas.urosario.edu.co/disertaciones/a.10127

Volumen 15, Número 1 / Enero-junio 2022

Versión PDF para imprimir desde

http://revistas.urosario.edu.co/index.php/disertaciones

15. Fernández, J. L. (2006). Guía de estilo sobre discapacidad para profesionales de los medios de comunicación. Ministerio de Trabajo y Estudios Sociales. http://sid.usal.es/idocs/F8/FD018497/medios_comunicacion.pdf

16. Francisco, S. (2003). Medios de Comunicación y Discapacidad. Un análisis de la cobertura periodística sobre inclusión social. Fundação Banco do Brasil; ANDI. https://www.andi.org.br/publicacao/ medios-de-comunicacion-y-discapacidad-una-analisis-de-la-cobertura-periodistica-sobre

17. Grinevald, C. (1998). Language endangerment in South America: A programmatic approach. In L. A. Grenoble \& L. J. Whaley (Eds.), Endangered Languages: Language Loss and Community Response (pp. 124-159). Cambridge University Press.

18. González, V. (s.f.). Medios y Discapacidad. ReDI, Red Por los Derechos de las Personas con Discapacidad. http://www.redi.org.ar/Documentos/Publicaciones/medios-y-discapacidad.pdf

19. Grupo Telefónica. (2005). Comunicación para todos. Pautas para la comunicación accesible. Grupo Telefónica. https://www.cermi.es/sites/default/files/docs/colecciones/ManualCXRATODOS_191005qxd.pdf

20. Harrison, D. K. (2008). When languages die: The extinction of the world's languages and the erosion of human knowledge. Oxford University Press.

21. Hermann, A., \& Steinbach, M. (Eds.). (2013). Nonmanuals in Sign Language. John Benjamins Publishing.

22. Hilger, A. I., Loucks, T. M., Quinto-Pozos, D., \& Dye, M. W. (2015). Second language acquisition across modalities: Production variability in adult L2 learners of American Sign Language. Second Language Research, 31(3), 375-388. https://doi.org/10.1177/0267658315570648

23. i Mimó, O. R. (1997). Declaración Universal de Derechos Lingüísticos. Revista Iberoamericana de Educación, 13, 281-289. https://doi.org/10.35362/rie1301146

24. Johnson, J. S., \& Newport, E. L. (1989). Critical period effects in second language learning: The influence of maturational state on the acquisition of English as a second language. Cognitive Psychology, 21(1), 60-99. http://dx.doi.org/10.1016/0010-0285(89)90003-0

25. Larsen, P. (2002). Mediated fiction. In K. B. Jensen (Ed.). (2020). A handbook of media and communication research: Qualitative and quantitative methodologies. Routledge.

26. Mayberry, R. (2010). Early language acquisition and adult language ability: What sign language reveals about the critical period for language. In M. Marschark \& P. Spencer, Oxford handbook of deaf studies, language, and education (pp. 281-291). Routledge.

27. Meir, I., Sandler, W., Padden, C., \& Aronoff, M. (2010). Emerging sign languages. In M. Marschark \& P. Spencer, Oxford handbook of deaf studies, language, and education (vol. 2) (pp. 267-280). Routledge.

28. Meléndez-Labrador, S. (2016). Comunicación interna incluyente: dos estudios de caso de inclusión laboral de personas con discapacidad auditiva en Bogotá. Investigación \& Desarrollo, 24(1), 26-52. https://www. redalyc.org/pdf/268/26846686002.pdf

29. Mides. (2018). Comunicación y discapacidad. Guía de buenas prácticas para trabajadoras y trabajadores de la comunicación. Ministerio de Desarrollo Social. http://pronadis.mides.gub.uy/innovaportal/ file/32256/1/comunicacion-y-discapacidad-guia-de-buenas-practicas-para-trabajadores-y-trabajadoras-de-la-comunicacion.pdf 


\section{DISERTACIONES}

ESTUDIOS

Grupos minoritarios y estigmatizados: diversidad funcional, religiosa, étnica, afectivo-sexual o de identidad de

30. Ministerio de Cultura de Perú. (2014). Comunicación que no discrimina. Guía para comunicadores. Ministerio de Cultura. http://repositorio.cultura.gob.pe/bitstream/handle/CULTURA/33/GUIA_2. pdf?sequence $=1 \&$ isAllowed=y

31. Nonaka, A. M. (2009). Estimating size, scope, and membership of the speech/sign communities of undocumented indigenous/village sign languages: The ban khor case study. Language \& Communication, 29(3), 210-229. http://dx.doi.org/10.1016/j.langcom.2009.02.004

32. Noseda, A. (2004). Pautas de estilo periodístico sobre discapacidad. Comité Federal de Radiodifusión (comFER). http://www.socieven.org/Content/biblio/Libro\%20Pautas\%20periodisticas\%20sobre\%20Discapacidad\%20enviado\%20por\%20Alejandra\%20Grzona.pdf

33. onu. (2006). Convención sobre los derechos de las personas con discapacidad. Naciones Unidas. https:// www.un.org/spanish/disabilities/default.asp?id=497

34. onu. (2015). Transforming our world: the 2030 Agenda for Sustainable Development. Division for Sustainable Development Goals.

35. Ortiz, I. D. L. R. R. (2005). Comunicar a través del silencio: las posibilidades de la lengua de signos española (Vol. 5). Universidad de Sevilla.

36. Peruzzo, C. (2020). Matrizes epistemológicas da comunicação popular e comunitária [Ponencia]. xv Congreso de la Asociación Latinoamericana de Investigadores de la Comunicación (ALAIC), Medellín.

37. Pfau, R., Steinbach, M., \& Woll, B. (Eds.). (2012). Sign language: an international handbook. De Gruyter Mouton.

38. Plaza, C. (2012). Deaf education and bilingualism. En R. Pfau, M. Steinbach \& B. Woll (Eds.), Sign language: an international handbook. De Gruyter Mouton.

39. Ponce, A. (2011). Diferentes: guía ilustrada sobre la diversidad y la discapacidad. Fundación Adecco; oHL. http://issuu.com/anundis/docs/diferentes-_gu__a_ilustrada_sobre_l?e=6720722/3776583

40. Ramírez, I. (2015). Comunicación para la inclusión. Manualdeestilo para el abordaje periodístico de la discapacidad en Venezuela. [Archivo PDF]. https://issuu.com/indipaper/docs/manual_comunicaci_n_para_la_inclus

41. Rascón, A. (2020). Manual de comunicación y discapacidad. Pontificia Universidad Católica de Chile; Centro uc Tecnologías de Inclusión CEDETI; CIAPAT. http://www.cedeti.cl/wp-content/uploads/2020/09/Manual-Comunicacio\%CC\%81n-y-Discapacidad-CEDETi-UC.pdf

42. Real Live Media. (2004). Guía para autogestores. Hacer que tu historia se escuche. Fenacerci. http://www. fenacerci.pt/realivemedia/espanol_easy/tools/guia_autogestores.pdf

43. Revilla, O., \& Carreras, O. (2018). Accesibilidad web. wCAG 2.1 de manera sencilla. [Archivo PDF]. https://www. usableyaccesible.com/archivos/Accesibilidad_Web_WCAG_21\%20ARIA_21_12_2020.pdf

44. Saldarriaga, F. (2014). Guía para entidades públicas servicio y atención incluyente. DNP; PNSc; Departamento Administrativo de la Función Pública; Arquitectura e interiores; Fundación Saldarriaga Concha. https://colaboracion.dnp.gov.co/CDT/Programa\%20Nacional\%20del\%20Servicio\%20al\%20Ciudadano/ Guia\%20Servicio\%20y\%20Atenci\%C3\%B3n\%20Incluyente.pdf

45. Sánchez de Amo, A., \& Marqués, S. (2002). Cómo mejorar la comunicación social sobre discapacidad. Guía práctica para entidades. Ministerio de Trabajo y Asuntos Sociales. http://www.siis.net/docs/ficheros/200406220024_24_0.pdf 


\section{DISERTACIONES}

Grupos minoritarios y estigmatizados: diversidad funcional, religiosa, étnica, afectivo-sexual o de identidad de género en la comunicación

ISSN: $1856-9536$

Doi: https://doi.org/10.12804/revistas.urosario.edu.co/disertaciones/a.10127

Volumen 15, Número 1 / Enero-junio 2022

Versión PDF para imprimir desde

http://revistas.urosario.edu.co/index.php/disertaciones

46. Universidad Católica Boliviana "San Pablo". (s.f.). Guía de orientaciones para la atención de personas con discapacidad en servicios públicos, privados y de interés social. [Archivo PDF]. https://avp.prenatal.tv/pluginfile.php/32283/mod_data/content/3400/2015-M4-Guia_de_orientaciones_para_la_atencion_de_personas_con_discapacidad_-_EMP.pdf

47. Vico, B. P., \& Heras, E. C. (2017). Inclusión del alumno con discapacidad auditiva en el aula de educación especial. Voces de la Educación, 2(4), 112-121. https://hal.archives-ouvertes.fr/hal-02534365/

48. Universidad Católica Boliviana "San Pablo". (2013). Manual sobre comunicación y discapacidad: el compromiso desde la información. soIPA. https://www.incluyeme.com/wp-content/uploads/2015/07/MANUAL-SOBRE-COMUNICACION-Y-DISCAPACIDAD.pdf

49. Werneck, C. (2005). Manual sobre desarrollo inclusivo: para los medios y profesionales de la comunicación. WVA Editora.

50. Zeshan, U., \& Dikyuva, H. (2013). Documentation of endangered sign languages: The case of Mardin Sign Language. Keeping Languages Alive: Documentation, Pedagogy and Revitalization, 29-41. 